\title{
Laminins and Nidogens in the Pericellular Matrix of Chondrocytes
}

\section{Their Role in Osteoarthritis and Chondrogenic Differentiation}

\author{
Boris Schminke, ${ }^{*}$ Jenny Frese,${ }^{\dagger}$ Christa Bode, ${ }^{*}$ Mary B. Goldring,${ }^{\ddagger}$ and Nicolai Miosge*
}

From the Department of Prosthodontics, * Tissue Regeneration Work Group, and the Clinic of Nephrology and Rheumatology, ${ }^{\dagger}$ Georg August University, Göttingen, Germany; and the Tissue Engineering, Regeneration, and Repair Program, ${ }^{\ddagger}$ Hospital for Special Surgery, Department of Cell and Developmental Biology, Weill Cornell Medical College, New York, New York

Accepted for publication

October 2, 2015.

Address correspondence to Nicolai Miosge, M.D., Ph.D., Robert Koch Strasse 40, 37075 Göttingen, Germany E-mail: nmiosge@gwdg.de.

\begin{abstract}
The aim of this study was to investigate the role of laminins and nidogen-2 in osteoarthritis (OA) and their potential to support chondrogenic differentiation. We applied immunohistochemistry, electron microscopy, siRNA, quantitative RT-PCR, Western blot, and proteome analysis for the investigation of cartilage tissue and isolated chondrocytes in three-dimensional culture obtained from patients with late-stage knee $0 \mathrm{~A}$ and nidogen-2 knockout mice. We demonstrate that subunits of laminins appear in $\mathrm{OA}$ cartilage and that nidogen-2-null mice exhibit typical osteoarthritic features. Chondrogenic progenitor cells (CPCs) produced high levels of laminin- $\alpha 1$, laminin- $\alpha 5$, and nidogen- 2 in their pericellular matrix, and laminin- $\alpha 1$ enhanced collagen type II and reduced collagen type I expression by cultured CPCs. Nidogen-2 increased SOX9 gene expression. Knockdown of nidogen-2 reduced SOX9 expression, whereas it up-regulated RUNX2 expression. This study reveals that the influence of the pericellular matrix on CPCs is important for the expression of the major regulator transcription factors, SOX9 and RUNX2. Our novel findings that laminins and nidogen-2 drive CPCs toward chondrogenesis may help in the elucidation of new treatment strategies for cartilage tissue regeneration. (Am J Pathol 2016, 186: 410-418; http://dx.doi.org/10.1016/j.ajpath.2015.10.014)
\end{abstract}

Articular cartilage is a connective tissue with chondrocytes embedded in a framework of collagens that consists primarily of collagen type II. ${ }^{1}$ Proteoglycans, ${ }^{2}$ such as aggrecan, ${ }^{3}$ and glycoproteins, such as cartilage oligomeric matrix protein (COMP), ${ }^{4}$ are associated with the collagen fibrils and act to stabilize the extracellular matrix (ECM). This unique structural organization is responsible for the biomechanical properties of cartilage, including tensile strength and resistance to compression and shear stress. ${ }^{5}$ Therefore, articular cartilage is responsible for the smooth transmission of forces within joints, allowing for painless skeletal movements. ${ }^{6}$ Chondrocytes in healthy articular cartilage rely on cellmatrix interactions ${ }^{1,7}$ (eg, via integrins). ${ }^{8}$

Disturbed cell-matrix interactions play an important role during the initiation of osteoarthritis (OA), leading to the loss of the superficial cartilage zone. Eventually, deep surface fissures, ECM degradation, collagen fiber fibrillation, and collagen type I accumulation occur. ${ }^{9}$ Furthermore, chondrocyte clusters are observed during the late stages of OA. ${ }^{10}$ The imbalance between cartilage degradation and matrix synthesis ultimately results in the complete loss of joint function. ${ }^{6}$ Current therapeutic interventions for $\mathrm{OA}$ provide only short-term symptomatic relief, and almost all patients ultimately require joint replacement. ${ }^{11}$ Adult articular cartilage exhibits a low capacity for regeneration, and ECM degradation overrides attempts of the resident chondrocytes to repair the matrix. There is evidence that chondrogenic progenitor cells (CPCs) drive the endogenous regenerative processes in cartilage. ${ }^{12}$

The basement membrane, a thin layer of condensed extracellular components, is composed primarily of four

Supported by German Research Foundation grant (Mi 5731A0-A).

Disclosures: None declared. 
major macromolecules (ie, laminins, nidogens, collagen type IV, and perlecan). Laminins are a trimer consisting of one of five $\alpha$, one of four $\beta$, and one of three $\gamma$ chains. ${ }^{13}$ Although cartilage does not contain a basement membrane, these molecules are present in the chondrocyte pericellular matrix (PCM), ${ }^{14}$ especially nidogens,${ }^{15}$ which are involved in OA pathogenesis. Furthermore, laminins and nidogens exert crucial roles during embryogenesis. ${ }^{16,17}$

The influence of laminins and nidogens on the chondrogenic potential of progenitor cells is not known. Herein, we demonstrate the roles of laminins and nidogen- 2 in OA cartilage and elucidate the influence of these basement membrane components on chondrogenic differentiation. Our investigation of laminins and nidogens in the PCM of chondrocytes may aid in the development of new treatment strategies, especially for tissue regeneration.

\section{Materials and Methods}

\section{Tissue Sources}

Cartilage samples were classified histopathologically by Osteoarthritis Research Society International standards ${ }^{18}$ and prepared as described elsewhere. ${ }^{12}$ Briefly, from our collection of $>500$ specimens during the past 15 years, we applied samples from the lateral condyle of knee joints collected from a region directly adjacent to the main defect with grade 4.0 to 4.5 , according to Pritzker et $\mathrm{al}^{18}$ (Supplemental Figure S1). The samples exhibited deep surface fissures and chondrocyte clusters and were only composed of the deep middle and the deep zones. This relates to former Mankin grading IV. The patients $[n=15$ ( 7 males and 8 females)] with a mean age of 67.1 (range, 44 to 85) years met the American College of Rheumatology classification ${ }^{19}$ and gave their written informed consent, consistent with the ethics regulations of our institution. Healthy specimens from three accident victims revealed macroscopically and histologically intact hyaline cartilage with a smooth surface, and all of the layers visible $^{18}$ corresponding to grade 0 to 1 , according to Pritzker et al $^{18}$ (Supplemental Figure S1). These samples were also from the taken lateral condyle of knee joints to match the OA samples. The animals were obtained according to the regulations of the animal welfare act of the County of Lower Saxony. The generation and genotyping of nidogen-2-null mice have been described earlier. ${ }^{20} \mathrm{We}$ used 9-week-old mice (young, $n=30$ ) and 24-week-old (adult, $n=30$ ) mice. The cartilage specimens were graded according to Glasson et $\mathrm{al}^{21}$ and exhibited grade 0.5 to 1 .

\section{Antibodies}

A monoclonal rat antibody was prepared against laminin$\alpha 1 .^{22}$ The polyclonal rabbit laminin- $\alpha 5$ antibody was a kind gift from the late Dr. Rupert Timpl (Max Planck Institute, Martinsreid, Germany). Monoclonal mouse anti-RUNX2 (sc-101145; Santa Cruz Biotechnology, Dallas, TX) and polyclonal rabbit anti-SOX9 (AP06583PU-N; Acris, Aachen, Germany) antibodies were purchased. A monoclonal mouse anti- $\alpha$-tubulin antibody (T6199; SigmaAldrich, St. Louis, MO) served as a loading control on immunoblots. Monoclonal antibody, anti-SMAD2/3 (c-8, sc-133098) was purchased from Santa Cruz Biotechnology. Polyclonal anti-phospho-SMAD2 (Ser465/467) was from Merck Millipore (Darmstadt, Germany). Appropriate secondary antibodies were obtained from Dako (Hamburg, Germany) and Sigma-Aldrich (St. Louis, MO).

\section{Tissue Fixation and Preparation}

Tissue samples were fixed in $4 \%$ paraformaldehyde in phosphate-buffered saline (PBS; freshly prepared), $\mathrm{pH} \mathrm{7.4,} \mathrm{at}$ $4^{\circ} \mathrm{C}$ overnight. Tissue samples were decalcified with buffered EDTA. Samples for light microscopy were dehydrated, embedded in paraffin, and divided into sections ( $6 \mu \mathrm{m}$ thin) using a Biocut microtome (Leica Instruments, Wetzlar Germany). For ultrastructural analysis, human cartilage specimens ( $1 \mathrm{~mm}^{3}$ thin) were embedded in LR-Gold (Polysciences, Warrington, PA) and mouse cartilage specimens in Epon (Polysciences), according to standard procedures. Ultrathin sections were cut using an ultramicrotome (Reichert-Jung, Wetzlar, Germany) and collected on nickel grids coated with Formvar (Agar Scientific, Wetzlar, Germany).

\section{Histochemistry}

Toluidine blue $\mathrm{O}$ (chemical identification number 52040) staining was performed with a toluidine blue dye solution ( $0.1 \mathrm{~g}$ toluidine blue dissolved in $100.0 \mathrm{~mL}$ distilled water) for 15 minutes at room temperature, rinsed several times with water, and exposed to $96 \%$ ethanol for 1 minute. Slides are dehydrated in absolute ethanol, cleared in xylene, and mounted in resinous medium.

\section{Light Microscopic Immunohistochemistry}

Immunoperoxidase staining was performed on paraffinembedded tissue sections, according to the protocol published elsewhere. ${ }^{12}$ Briefly, tissue samples were deparaffinized, rehydrated, and rinsed for 10 minutes in PBS. Endogenous peroxidase was blocked for 45 minutes with $3 \% \mathrm{H}_{2} \mathrm{O}_{2}$ /methanol in the dark. Each reaction was followed by rinsing for 10 minutes in PBS. The sections were pretreated for 5 minutes with $10 \mu \mathrm{g} / \mathrm{mL}$ protease XXIV (Sigma-Aldrich) and chondroitinase (SigmaAldrich). The antibodies were applied at a dilution of 1:100 in PBS for 12 hours at room temperature. A standard peroxidaseantiperoxidase procedure was followed, and a peroxidasecoupled goat-anti-rabbit (Dako) or goat anti-rat antibody (Dako) was applied at a dilution of 1:150 in PBS for 1 hour at room temperature. The color reaction was performed using a diaminobenzidine substrate (Sigma-Aldrich).

Negative controls were performed by treating the sections with swine serum instead of the primary antibody for the 
polyclonal antibodies and isotype matched IgGs for the monoclonal mouse antibody. Furthermore, color reactions were performed without prior antibody treatment. All experimental data are representatives of five individual immunoreactions.

\section{Electron Microscopic Immunohistochemistry}

Ultrathin tissue sections were incubated with anti-laminin- $\alpha 1$ and anti-laminin- $\alpha 5$ antibodies, diluted 1:100 in PBS for 16 hours at room temperature, as published previously. ${ }^{12}$ An affinity-purified goat anti-rabbit $\operatorname{IgG}$ and a goat anti-rat antibody (Medac, Wedel, Germany) were coupled to 16-nm colloidal gold particles, according to our standard protocols. ${ }^{15}$ The appropriate secondary gold-coupled antibodies, diluted 1:300 in PBS, were applied for 20 minutes at room temperature. Staining with uranyl acetate followed, and reactions were examined using a Zeiss (Jena, Germany) EM 906E electron microscope. The quantification of the gold particles in the PCM was performed in five OA patient samples and three healthy samples. Nine PCMs per cell type and patient were quantified. For the control of the immunogold histochemistry, the tissue samples were incubated with pure gold solution to exclude unspecific binding of free colloidal gold. Furthermore, the reactions were performed with gold-coupled goat anti-rabbit IgG to exclude non-specific IgG binding.

\section{Cell Isolation and Culture}

The method for obtaining CPCs and chondrocytes from cartilage tissue was described previously. ${ }^{12}$ Standard explant cultures were performed with 8 - to $15-\mathrm{mm}^{3}$ tissue specimens taken from cartilage tissues described above. Care was taken to ensure that no bone tissue was included. Outgrown CPCs were harvested after 10 days, and $10^{3}$ cells $/ \mathrm{cm}^{2}$ were transferred to cell culture in Dulbecco's modified Eagle's medium (Invitrogen, Darmstadt, Germany) with 10\% fetal bovine serum (Invitrogen) supplemented with $50 \mu \mathrm{g} / \mathrm{mL}$ gentamicin and $10 \mathrm{mmol} / \mathrm{L} \mathrm{L}$-glutamine. Chondrocytes from healthy and OA cartilage were harvested after digestion for 6 hours at $37^{\circ} \mathrm{C}$ with the aid of $152 \mathrm{U} / \mathrm{mL}$ collagenase I (Invitrogen), $280 \mathrm{U} / \mathrm{mL}$ collagenase II (Merck Millipore), and $15 \mathrm{U} / \mathrm{mL}$ dispase (Invitrogen). The digested material was filtered through a $40-\mu \mathrm{m}$ mesh sieve (BD Falcon, Mannheim, Germany) to eliminate cell-matrix residues. To test the influence of the PCM components, 40,000 cells in P2 were mixed in $20 \mu \mathrm{L}$ of $1.2 \%$ alginate solution supplemented with $125 \mathrm{ng} / \mathrm{mL}$ of either laminin (Merck Millipore) or nidogen-2 (a kind gift from the late Roswitha Nischt, Cologne, Germany) per bead ${ }^{23}$ and grown in Dulbecco's modified Eagle's medium for 4 weeks in three-dimensional alginate beads in 6 -well plates (10 beads per well).

\section{RNA Extraction and cDNA Synthesis}

Cells $\left(1 \times 10^{6}\right)$ were directly dissolved in $0.5 \mathrm{~mL}$ RLT buffer, and the RNA was isolated using the RNeasy Mini
Kit (Qiagen, Hilden, Germany), according to the manufacturer's instructions. RNA was reverse transcribed into cDNA using the QuantiTect Reverse Transcription Kit (Qiagen).

\section{Quantitative RT-PCR}

The preparation of the master-mix, primer design, and PCR settings were already published. ${ }^{12}$ The primer sequences are the following: aggrecan, $5^{\prime}$-ACAGCTGGGGACAT- $3^{\prime}$ (forward) and 5'-GTGGAATGCAGAGG-3' (reverse); collagen (COL) 1A1, 5'-TTCCCCCAGCCACAAAGAGTC- $3^{\prime}$ (forward) and $5^{\prime}$-CGTCATCGCACAACACCT-3' (reverse); COL2A1, 5'-CTCCTGGAGCATCTGGAGAC$3^{\prime}$ (forward) and 5'-ACCACGATCACCCTTGACTC-3' (reverse); and SOX9, $5^{\prime}$-CAGGCTTTGCGATTTAAGGA$3^{\prime}$ (forward) and 5'-CCGTTTTAAGGCTCAAGGTG-3' (reverse). The relative ratios were calculated according to Pfaffl. ${ }^{24}$ Gels were prepared when necessary by dissolving $1.5 \%$ agarose in $50 \mathrm{~mL}$ of Tris-EDTA buffer by boiling. After brief cooling, $3.5 \mu \mathrm{L}$ Roti-GelStain (Carl Roth, Karlsruhe, Germany) was added to visualize DNA. We used $20 \mu \mathrm{L}$ of the PCR product and mixed it with loading buffer. PCR product size was determined using a GeneRuler 100-bp DNA Ladder (Thermo Scientific, Darmstadt, Germany).

\section{siRNA Transfection}

We harvested $5 \times 10^{5} \mathrm{CPCs}$ and resuspended the cells in $100 \mathrm{~mL}$ of Human Chondrocyte Nucleofector Solution (Lonza) with $10 \mathrm{~mL}$ siRNA of nidogen-2 at $0.2 \mathrm{nmol}$ (Qiagen). We used pMAX-GFP (Lonza, Basel, Switzerland) as a positive control for transfection and a scrambled RNA, AllStars-negative siRNA (Qiagen) as a negative control. We selected the U23 program in the Nucleofector $2 b$ Device (Lonza), mixed the samples with $500 \mu \mathrm{L}$ medium, and immediately transferred the solution to each of the six preincubated wells containing $1 \mathrm{~mL}$ of medium. The culture medium was replaced the next day to remove dead cells. The cells were harvested 48 hours after transfection.

\section{Immunoblotting}

A total of $1.5 \times 10^{5} \mathrm{CPCs}$ were lysed in $30 \mu \mathrm{L}$ of $3 \times \mathrm{SDS}$ with $10 \% \beta$-mercaptoethanol and heated at $95^{\circ} \mathrm{C}$ for 5 minutes. SDS-PAGE was performed using $6 \%$ acrylamide in the stacking gel and $8 \%$ in the separation gel. After separation by electrophoresis, the proteins were blotted on an Immobilon-P Transfer-Membrane (Merck Millipore) and stained with Coomassie Blue. After destaining and blocking, the primary antibodies were dissolved in 5\% milk in Tris-buffered saline and Tween 20 and incubated for 12 hours at $4{ }^{\circ} \mathrm{C}$. Visualization of the proteins was achieved via the application of Western Bright Sirius horseradish peroxidase substrate (Advansta, Menlo Park, CA). 


\section{Proteome Analysis}

Gel bands were cut and washed with water, reduced with dithiothreitol, and alkylated with iodoacetamide. Gel spots were digested twice overnight with trypsin NB Premium Grade, MS approved from porcine pancreas (Serva, Ingelheim, Germany). Peptide extraction was performed using aqueous acetonitrile, and mass spectrometric analysis was performed, as published previously. ${ }^{25}$

\section{Statistical Analysis}

Statistical product and service solutions software version 13.0 was used (IBM, Ehningen, Germany). The observed data were tested, and the representative data shown are the means and SD of at least three different samples, if not stated otherwise. After testing for normality of distribution and homogeneity of variances, we performed analysis of variances and post hoc pairwise comparisons of the mean values. The Pearson correlation coefficients were calculated to examine the relationships between parameters. $P<0.05$ was considered significant.

\section{Results}

Differential Expression of Laminin- $\alpha 1$ and Laminin- $\alpha 5$ in Human Cartilage Tissue

Laminin- $\alpha 1$ and laminin- $\alpha 5$ (Figure 1) were detected in healthy and OA cartilage specimens. Although we observed a small amount of laminin- $\alpha 1$ and less laminin- $\alpha 5$ in healthy cartilage (Figure 1A), we detected significantly more laminin- $\alpha 1$, as well as laminin- $\alpha 5$, in OA cartilage (Figure 1B). This was corroborated by quantification of the immunohistochemistry (Figure 1, C and D).

We applied gold-coupled antibodies to compare and quantify the amount of laminins in healthy chondrocytes, OA chondrocytes, and CPCs. In healthy cartilage, chondrocytes were typically round in morphology, with only a few 20-nm gold particles in the PCM, whereas OA cartilage exhibited marked gold staining for laminin- $\alpha 1$ in the PCM (Figure 2A). However, the most intense staining for laminin- $\alpha 1$ was detected in the PCM (Figure 2A) of the CPCs. Staining for laminin- $\alpha 5$ was similar (Figure 2B). Quantification of the gold particles showed significant differences in laminin- $\alpha 1$ in the PCM of healthy chondrocytes, OA chondrocytes, and CPCs (Figure 2C). Laminin- $\alpha 5$ staining in the PCM differs significantly between the healthy and OA chondrocytes on the one hand and CPCs on the other (Figure 2C).

\section{Effect of the Lack of Nidogen-2 on Mouse Cartilage Tissue}

Wild-type 9-week-old mice exhibited intense toluidine blue staining, indicating normal proteoglycan content (Figure 3A); however, even at this age, the nidogen-2 knockout (KO) mice showed less intense staining in the deeper zones, indicative of
A

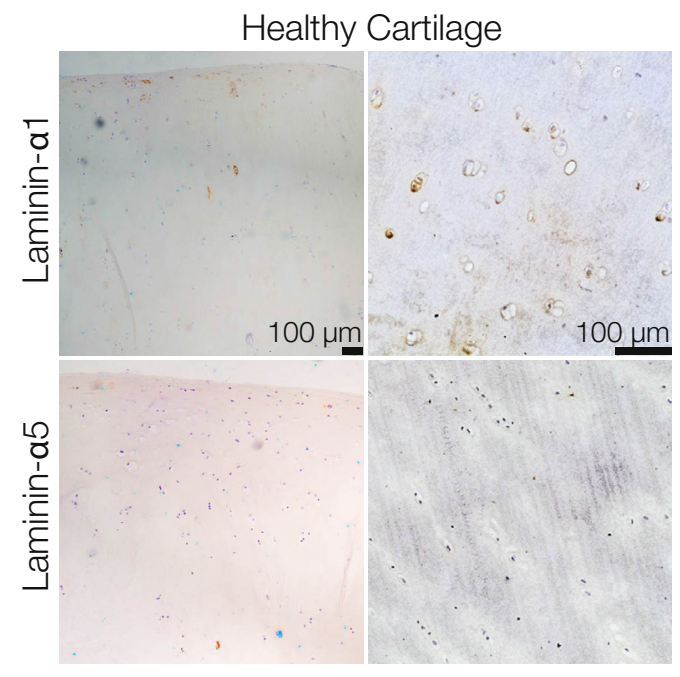

B

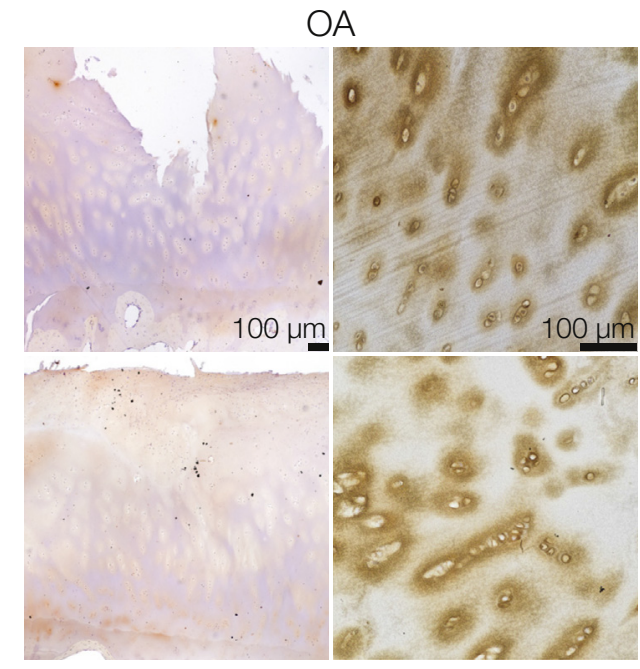

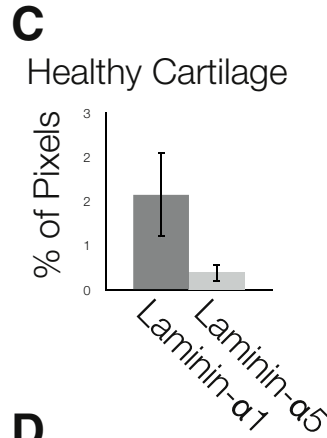

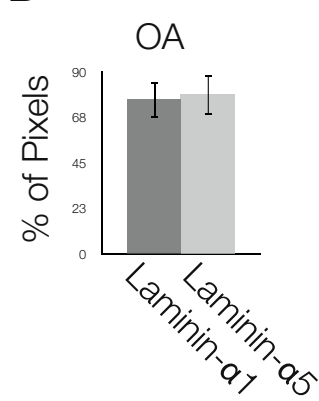

Figure 1 In the immunohistochemical staining overview of healthy cartilage, sparse staining is seen for laminin- $\alpha 1$ mainly in the upper zones (A, top right panels), whereas nearly no reaction is seen for laminin- $\alpha 5$ (A, bottom left panels). The same pattern is seen in the higher magnifications. The osteoarthritis ( $0 \mathrm{~A}$ ) cartilage of the lateral condyle of the knee joint (0steoarthritis Research Society International grade 4.5) only exhibits the deep middle and the deep zones. B: This relates to former Mankin grading IV. Top panels: Herein, in the 0A cartilage, enhanced immunoreactions of laminin- $\alpha 1$, notably within the pericellular matrix of chondrocyte clusters, were detected. Bottom panels: The same was seen for laminin- $\alpha 5$. Quantification of the staining intensity in healthy cartilage (C) and in OA cartilage (D). Data denote the means \pm SD; $n=5$ individual experiments (C and D). Scale bars, $100 \mu$ m. 

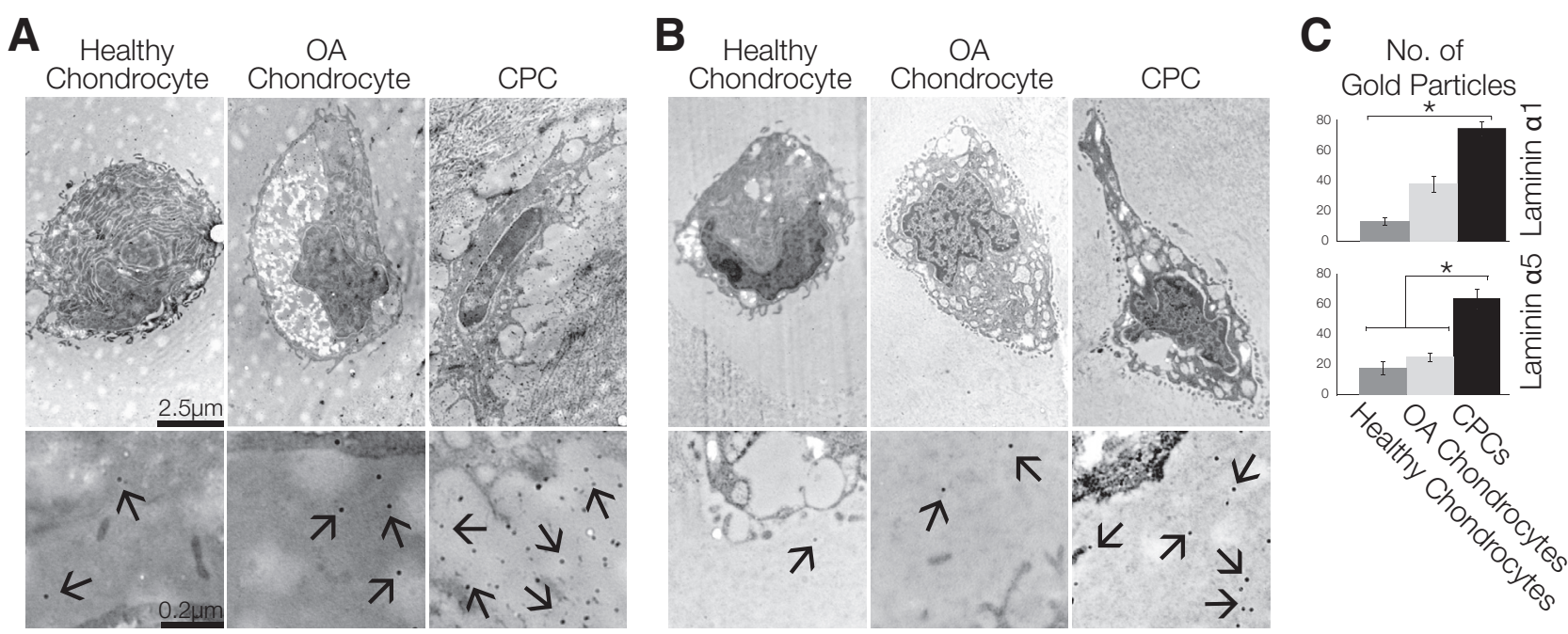

Figure 2 Analysis of laminin- $\alpha 1$ in the pericellular matrix (PCM) of chondrogenic cells. A: The PCM of a healthy chondrocyte with rounded morphology has only a few 20-nm gold particles (arrows), representing the protein. 0steoarthritis (OA) chondrocytes are associated with more laminin- $\alpha 5$ in the PCM. Interestingly, the elongated chondrogenic progenitor cells (CPCs) of late-stage $0 \mathrm{~A}$ show more laminin- $\alpha 1$ in the PCM compared with healthy and 0A chondrocytes. B: The same pattern as in A is seen for laminin- $\alpha 5$. C: Quantification of laminin- $\alpha 1$ and laminin- $\alpha 5$ in the PCM of healthy and $0 A$ chondrocytes and CPCs. Data denote the means \pm SD (C). $n=3$ individual experiments (C). ${ }^{*} P \leq 0.05$. Scale bar $=2.5 \mu \mathrm{m}$ (A and $\mathbf{B}$, top panels); $0.2 \mu \mathrm{m}(\mathbf{A}$ and $\mathbf{B}$, bottom panels).

increased calcified cartilage (Figure 3A). In 24-week-old mice, the differences in staining patterns were more pronounced in wild-type mice, compared with KO mice (Figure 3A), where there was a highly reduced proteoglycan content in both the calcified cartilage adjacent to the subchondral bone and in the remaining articular cartilage. Quantification of the width of the calcified cartilage zone revealed a wider zone in the $\mathrm{KO}$ mice (Figure 3B), and the reduction of the proteoglycan content was most pronounced in the 24-week-old $\mathrm{KO}$ mice (Figure 3C). The hyaline cartilage of old KO mice knee joints exhibited signs of early calcification, which is also known to play a role during the pathogenesis of $\mathrm{OA}^{26}$

\section{Chondrogenic Roles of Laminins and Nidogen-2}

Chondrocytes from healthy cartilage and OA cartilage were cultured in alginate beads, which simulates a threedimensional environment, ${ }^{23}$ and incubated with laminin or nidogen-2 for 4 weeks. The levels of aggrecan mRNA did not change with nidogen-2 treatment in healthy chondrocytes, but the lower level in OA chondrocytes was significantly increased to that observed in healthy chondrocytes (Figure 4A). In contrast, the levels of SOX9 mRNA were significantly increased in healthy chondrocytes by nidogen-2 to the levels observed in OA chondrocytes, which did not change on treatment with nidogen-2 (Figure 4A). COL2Al and COL1Al gene expression patterns (Figure 4A) were similar in healthy and OA chondrocytes and not influenced significantly by nidogen-2 treatment. In contrast, laminin supplementation significantly increased the COL2A1 mRNA levels in healthy and OA chondrocytes and reduced COL1A1 expression (Figure 4B). Herein, the levels of aggrecan and SOX9 mRNA were not influenced significantly by laminin treatment (Figure 4B). Our experiments using CPCs derived from diseased OA tissue showed significantly increased levels of aggrecan and SOX9 mRNA (Figure 4C) after nidogen-2 stimulation. However, laminin increased COL2A1 mRNA and reduced COL1A1 mRNA (Figure 4D) compared with untreated CPCs. These results indicate a change in the gene expression signature toward chondrogenesis in response to laminin and nidogen-2, at least in vitro.

The PCM is involved in cartilage calcification and is permissive for the SOX9-RUNX2 antagonism.

Interestingly, the ultrastructural analysis of nidogen-2 $\mathrm{KO}$ mice cartilage right above the tidemark indicated that calcium phosphate crystals in the ECM, a common phenomenon in OA cartilage, ${ }^{27}$ were present at higher levels than in wild-type mice (Figure 5A). These findings were confirmed in an ex vivo three-dimensional experiment. In the nidogen$2 \mathrm{KO}$, a dense collagen-fiber framework occurs together with crystals, characteristic of bone tissue, whereas the wildtype cells exhibited regularly formed collagen fibrils without crystals (Figure 5A). Therefore, we hypothesized that nidogen-2 is involved in the regulation of the calcification process in chondrocytes. When nidogen-2 is not present in the PCM of the cartilage tissue, the chondrocytes change their gene expression toward an increased RUNX2 level and decreased SOX9 level, reducing their chondrogenic potential. Therefore, we evaluated the mRNA levels of nidogen-2 in healthy chondrocytes, OA chondrocytes, and CPCs. CPCs expressed more nidogen-2 mRNA than healthy or OA chondrocytes (Figure 5B), consistent with previous findings at the protein level. ${ }^{15}$ However, siRNA knockdown of nidogen-2 protein in CPCs resulted in increased RUNX2 and decreased SOX9 (Figure 5C). Equal loading of the gels 


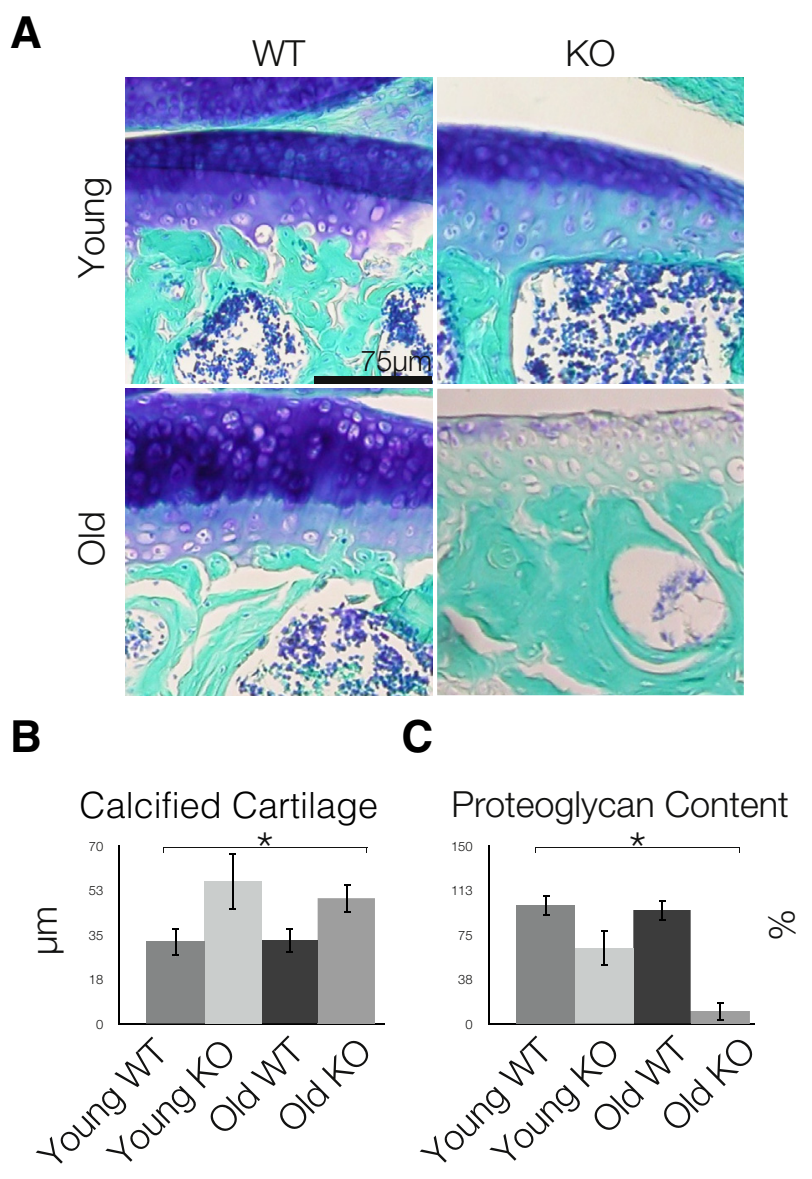

Figure 3 Nidogen-2-deficient mice, with toluidine blue staining of the joint surfaces. A: The wild-type (WT) joint surface exhibits a staining pattern well known for young mice, whereas their knockout (KO) counterparts exhibit reduced staining of the calcified cartilage zone. The 24-week-old WT mice show a staining pattern known for older mice compared with the much-reduced staining intensity in the older $\mathrm{KO}$ mice. B: Quantification reveals a significantly wider calcified cartilage zone (up to $50 \mu \mathrm{m})$ in young $\mathrm{KO}$ mice. C: The loss of proteoglycans as revealed by a reduced staining intensity for toluidine blue [number of blue pixels as counted via ImageJ (NIH, Bethesda, MD; http://imagej.nih.gov/ij)] is most pronounced in the older 24-week-old K0 mice. Data are means $\pm \mathrm{SD} ; n=3$ individual experiments (B and $\mathbf{C}$ ). ${ }^{*} P \leq 0.05$. Scale bar $=75 \mu \mathrm{m}$.

was confirmed using $\alpha$-tubulin (Figure 5C). After the knockdown of nidogen-2 and the subsequent increase in RUNX2, the protein expression signature of the CPCs shifted to reflect the osteoblastic, rather than the chondrogenic, lineage.

\section{Nidogen-2 Knockdown in CPCs Reduces pSMAD2}

The Coomassie Blue staining of protein extracts from CPCs after siRNA knockdown of nidogen-2 showed a band at approximately $70 \mathrm{kDa}$ that was more intensely stained than in the control (Figure 6A). Proteome analysis (Figure 6B) of these bands revealed four up-regulated candidate proteins: heat shock cognate $71-\mathrm{kDa}$ protein, heat shock 70$\mathrm{kDa}$ protein $1 \mathrm{~A} / 1 \mathrm{~B}, \mathrm{X}$-ray repair cross-complementing protein 6, and annexin A6. Heat shock cognate 71-kDa protein is known to interact with CITED1, which leads to inhibition of SMAD signaling. ${ }^{28}$ Herein, we show, for the first time, that nidogen-2, as a part of the PCM, is involved in this signaling pathway. The consequence of the nidogen-2 $\mathrm{KO}$ and the enhanced heat shock cognate 71-kDa protein levels could be because of a reduction of SMAD signaling. Indeed, when we performed a Western

A
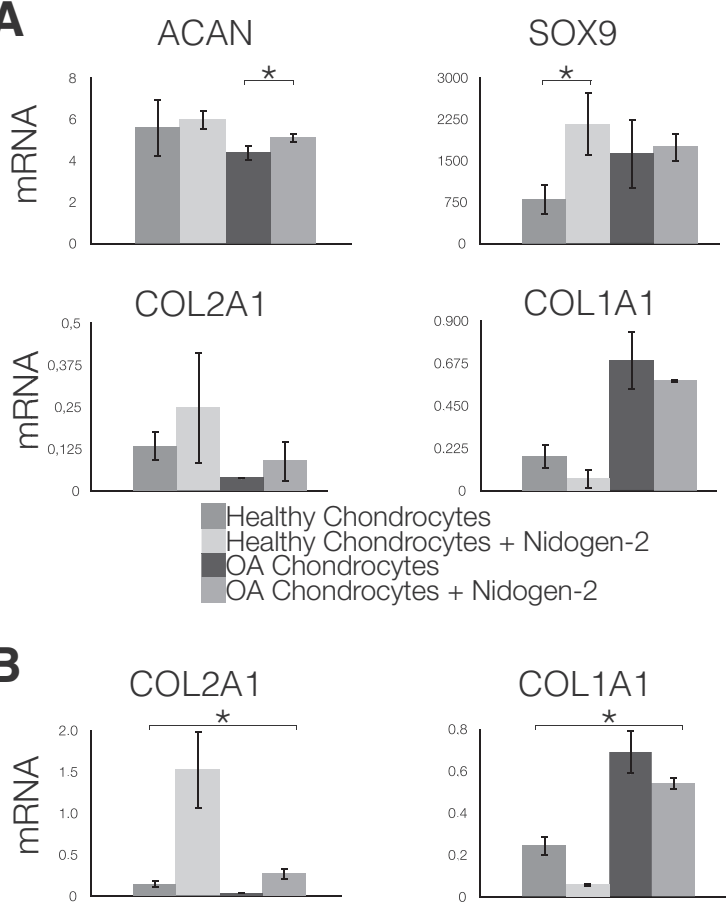

B
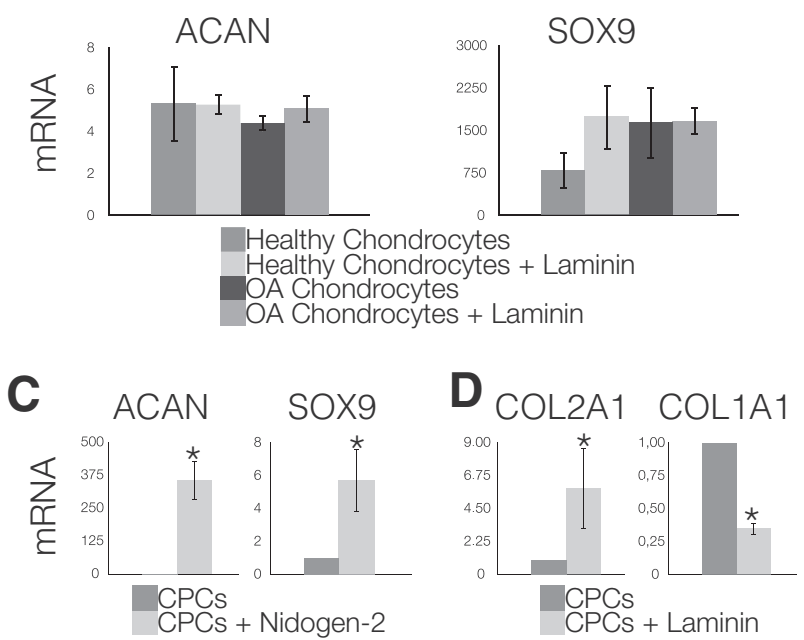

Figure 4 Chondrogenic roles of laminin and nidogen-2. A: Aggrecan (ACAN) and SOX9 [mRNA levels are significantly enhanced in chondrocytes by nidogen-2, whereas there is no difference in collagen (COL) 2A1 or COL1A1]. B: Chondrocytes stimulated with laminin exhibit a significantly increased expression of COL2A1 and decreased COL1A1. Laminin has no effect on ACAN or S0X9. In chondrogenic progenitor cells (CPCS), nidogen-2 significantly increases ACAN and SOX9 expression (C), whereas laminin significantly enhances COL2A1 and reduces COL1A1 (D). Data are means \pm SD and $n=3$ individual experiments for all panels. ${ }^{*} P \leq 0.05$. $0 \mathrm{~A}$, osteoarthritis. 
A

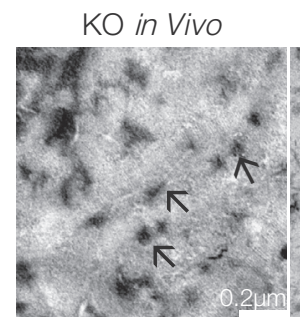

$\mathrm{KO}$ in Vitro

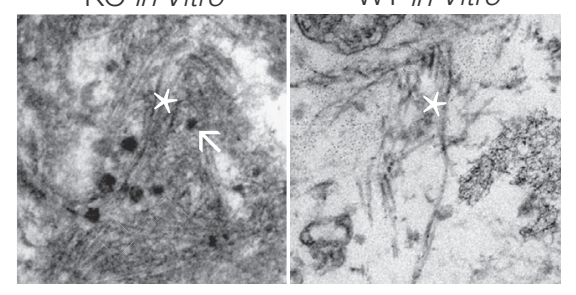

B

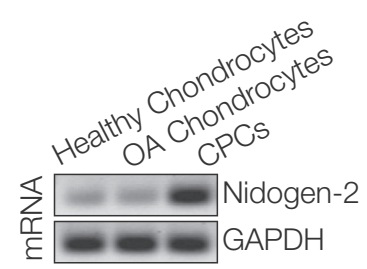

C

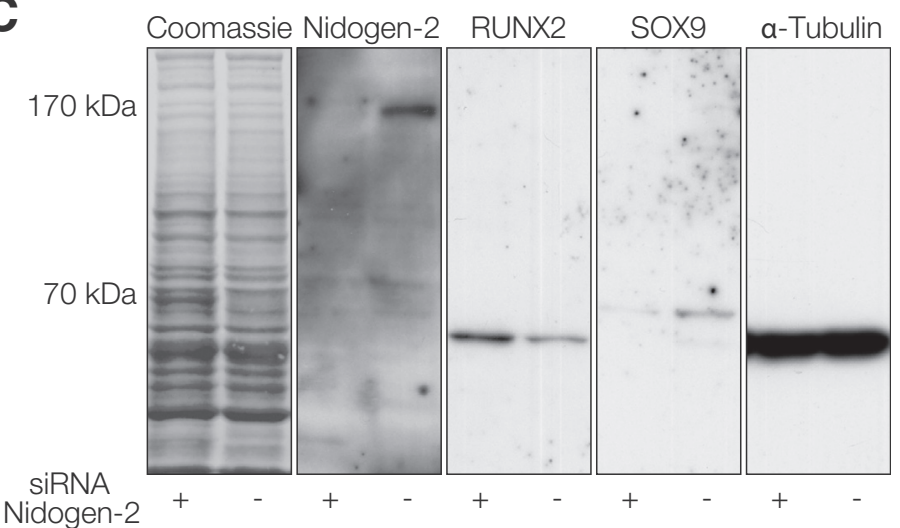

Figure 5 The pericellular matrix (PCM) is involved in cartilage calcification and the regulation of the SOX9-RUNX2 antagonism. A: Ultrastructural analysis of laminin knockout (KO) cartilage above the tidemark shows crystals (arrows) in the extracellular matrix (ECM) but not in wild-type (WT) cartilage. These findings were confirmed in ex vivo alginate bead experiments, where the KO cells produce crystals (arrows), which are embedded in the irregular collagen framework, whereas the WT cells are associated with regularly formed collagen fibrils. Arrows point at the crystals, whereas asterisks mark the ECM without these crystals. B: Quantitative RT-PCR shows that chondrogenic progenitor cells (CPCs) express more nidogen-2 mRNA than healthy and osteoarthritis (OA) chondrocytes. C: siRNA knockdown of nidogen-2: first lanes, Coomassie Blue staining reveals the proper separation of the proteins from the siRNA experiment and the control; second lanes, immunoblotting of nidogen-2 confirms the KO compared with the control, where there is increased RUNX2 expression (third lanes) and decreased SOX9 expression (fourth lanes); last lanes, equal loading of the gels was confirmed using $\alpha$-tubulin, with + for control and - for the K0. Scale bar $=0.2 \mu \mathrm{m}$.

blot for activated/phosphorylated SMAD2, we observed reduced pSMAD2 (Figure 6C).

\section{Discussion}

Chondrocytes from OA cartilage are known to express less SOX9, which may promote restoration of the ECM of OA tissue in tissue engineering approaches. ${ }^{29}$ Herein, we demonstrate that the addition of extracellular nidogen-2 enhanced SOX9 expression in CPCs, suggesting the importance of the molecular composition of the PCM. We show that the chondrogenic cells of $\mathrm{OA}^{30}$ could be influenced by nidogen- 2 and increase the ratio of collagen type II/collagen type I mRNA. We have already shown that SOX9 and RUNX2 can act as antagonists in chondrogenic
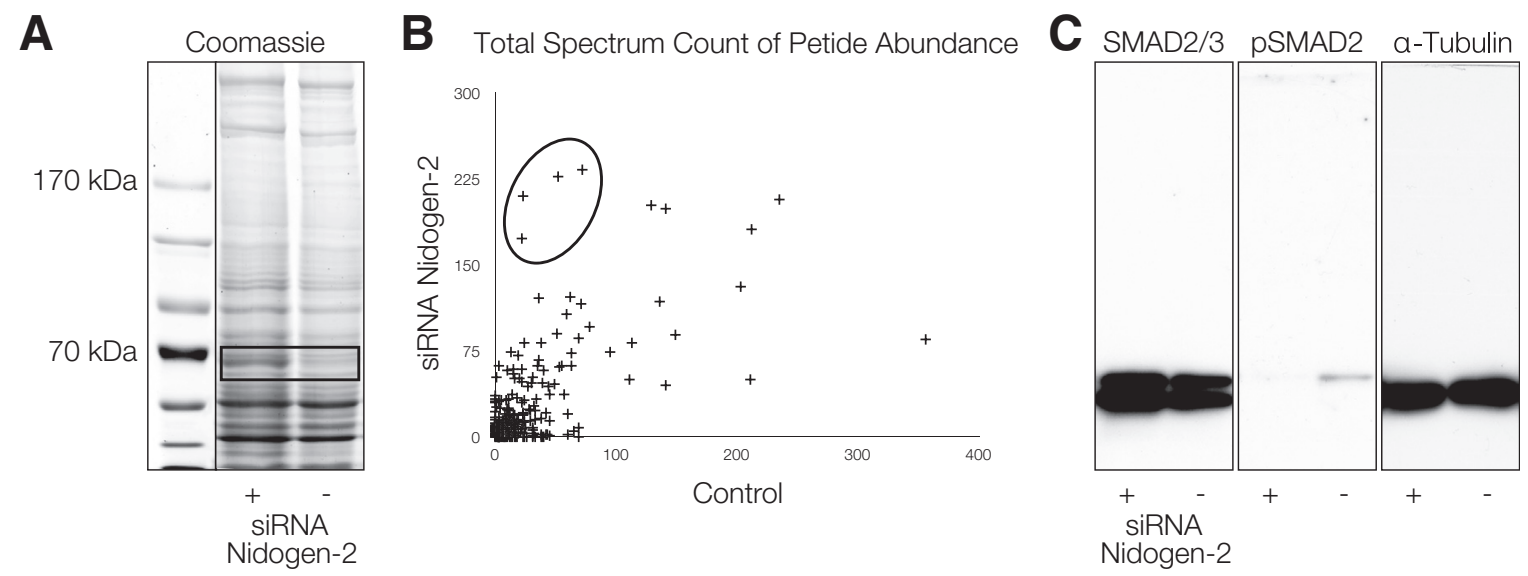

Figure 6 Nidogen-2 knockdown in chondrogenic progenitor cells (CPCs) reduces pSMAD2. A: Coomassie Blue staining of extracts from CPCs after siRNA knockdown of nidogen-2 compared with control. The staining shows a strong band at approximately $70 \mathrm{kDa}$ in nidogen-2 knockdown CPCs. B: Total spectrum shows four candidates, which are up-regulated after nidogen-2 knockdown: heat shock cognate $71-\mathrm{kDa}$ protein, heat shock 70-kDa protein $1 \mathrm{~A} / 1 \mathrm{~B}, \mathrm{X}$-ray repair cross-complementing protein 6, and annexin A6. C: Immmunoblots show reduced pSMAD2 expression after the nidogen-2 knockdown compared with control with no difference in total SMAD2 protein or in $\alpha$-tubulin as a loading control. 
cells. ${ }^{12}$ RUNX2 is a well-known osteogenic factor that also promotes terminal differentiation of hypertrophic chondrocytes, and it is enhanced in osteoarthritic cartilage. ${ }^{31}$ RUNX2 induces matrix metalloprotease (MMP) 13, which promotes OA progression. ${ }^{32}$ Herein, we show that the increased amount of SOX9, at least in chondrogenic cells in vitro, also depends on nidogen- 2 . The $\mathrm{KO}$ of nidogen- 2 reduces the expression of SOX9, whereas RUNX2 is enhanced. Furthermore, the CPCs express more nidogen-2 than healthy or OA chondrocytes in vivo or in vitro. Therefore, we speculate that nidogen-2 could have a regenerative influence on articular cartilage during OA.

Nidogen-2 has already been shown to exhibit a protective role in neoplastic processes, because deletion of nidogen-2 in mice increases lung metastasis on challenge with melanoma cells. ${ }^{33}$ Nidogen-2 is also important for the maintenance of the glomerular structure in the diseased kidney. ${ }^{34}$ Herein, we introduce a new role for nidogen-2 in cartilage biology. Consistent with these observations are findings that nidogen-2 is involved in the bone morphogenetic protein response and mitogen-activated protein kinase signaling cascades $^{35}$ and that mitogen-activated protein kinase 3 upregulates SOX9-promoting chondrogenesis. ${ }^{36}$ Our demonstration that the signaling of SMADs is promoted by nidogen-2 in CPCs adds another piece to the puzzle. This needs to be investigated in greater detail in the future.

Stem cells are influenced by the surrounding ECM, especially the basement membrane, ${ }^{37,38}$ and the ECM is also important for the stem cell niche. ${ }^{39}$ For example, laminin is a component of the human limbal epithelial stem cell niche. ${ }^{40}$ Recent studies have shown that laminin enhances the proliferation of mouse embryonic stem cells ${ }^{41}$ and prevents apoptosis in mouse myogenic cells. ${ }^{42}$ Our results showing enhanced laminin- $\alpha 1$ and laminin- $\alpha 5$ in the PCM of chondrocytes, including those in clusters in OA, suggest potential regenerative activity. The PCM would then act as a special form of basement membrane influencing progenitor cell behaviors, including proliferative capacity in the formation of clusters. ${ }^{10}$ Furthermore, we show that laminin promotes restoration of the chondrocyte phenotype, downregulating collagen type I and up-regulating collagen type II mRNAs.

On the other hand, some researchers see chondrocyte clusters as a sign of degeneration, with cells migrating together in the degraded ECM. ${ }^{10}$ In line, laminin may influence migration, which has been reported in three-dimensional cell culture studies, ${ }^{43}$ and laminin- $\alpha 5$ is a regulator of lymphocyte migration. ${ }^{44}$ The key players of OA are MMPs, which are responsible for the degradation of ECM, especially collagen type II-degrading MMP13, and simultaneous enhancement of migration. ${ }^{45} \mathrm{~A}$ recent study showed that MMP3, which digests proteoglycans and other noncollagen proteins, is controlled by the laminin- $\alpha 4$ subunit. ${ }^{46}$

In summary, the expression of nidogen-2 and laminin is increased in human OA cartilage, and they act as chondrogenic regulators, especially for CPCs. Furthermore, nidogen-2 influences the antagonism between RUNX2 and SOX9, increasing SOX9 and promoting collagen type II synthesis, consistent with a chondrogenic potential. Laminin also promotes chondrogenesis, enhancing collagen type II, COMP, and aggrecan expression, and down-regulating collagen type I. Therefore, these findings on laminin and nidogen-2 may aid in the elucidation of new treatment options, especially for tissue regeneration.

\section{Acknowledgments}

We thank the staff of the animal facilities at the Max Planck Institute for Experimental Medicine Göttingen and the Medical Faculty of the University of Göttingen for animal care; the late Dr. Rupert Timpl for providing the polyclonal rabbit laminin$\alpha 5$ antibody; and the late Dr. Roswitha Nischt for providing the nidogen-2 knockout mice and helping with the protein production. We dedicate this work to the late Dr. Nischt.

B.S. conceived the study, acquired, analyzed, and interpreted data, and performed statistical analysis; J.F. and C.B. acquired, analyzed, and interpreted data; M.B.G. interpreted the data and prepared the manuscript; N.M. conceived the study, interpreted data, and prepared the manuscript.

\section{Supplemental Data}

Supplemental material for this article can be found at http://dx.doi.org/10.1016/j.ajpath.2015.10.014.

\section{References}

1. Kuettner KE: Biochemistry of articular cartilage in health and disease. Clin Biochem 1992, 25:155-163

2. Iozzo RV, Schaefer L: Proteoglycan form and function: a comprehensive nomenclature of proteoglycans. Matrix Biol 2015, 42:11-55

3. Knudson CB, Knudson W: Hyaluronan-binding proteins in development, tissue homeostasis, and disease. FASEB J 1993, 7:1233-1241

4. Hedbom E, Antonsson P, Hjerpe A, Aeschlimann D, Paulsson M, Rosa-Pimentel E, Sommarin Y, Wendel M, Oldberg A, Heinegard D: Cartilage matrix proteins: an acidic oligomeric protein (COMP) detected only in cartilage. J Biol Chem 1992, 267:6132-6136

5. Sandell LJ: Modern molecular analysis of a traditional disease: progression in osteoarthritis. Arthritis Rheum 2007, 56:2474-2477

6. Buckwalter JA, Mankin HJ: Articular cartilage: degeneration and osteoarthritis, repair, regeneration, and transplantation. Instr Course Lect 1998, 47:487-504

7. Theocharis AD, Gialeli C, Bouris P, Giannopoulou E, Skandalis SS, Aletras AJ, Iozzo RV, Karamanos NK: Cell-matrix interactions: focus on proteoglycan-proteinase interplay and pharmacological targeting in cancer. FEBS J 2014, 281:5023-5042

8. Loeser RF: Integrins and cell signaling in chondrocytes. Biorheology 2002, 39:119-124

9. Poole AR: An introduction to the pathophysiology of osteoarthritis. Front Biosci 1999, 4:D662-D670

10. Poole CA: Articular cartilage chondrons: form, function and failure J Anat 1997, 191(Pt 1):1-13

11. Lohmander LS, Roos EM: Clinical update: treating osteoarthritis. Lancet 2007, 370:2082-2084

12. Koelling S, Kruegel J, Irmer M, Path JR, Sadowski B, Miro X, Miosge N: Migratory chondrogenic progenitor cells from repair tissue 
during the later stages of human osteoarthritis. Cell Stem Cell 2009, 4 324-335

13. Aumailley M, Bruckner-Tuderman L, Carter WG, Deutzmann R, Edgar D, Ekblom P, Engel J, Engvall E, Hohenester E, Jones JC, Kleinman HK, Marinkovich MP, Martin GR, Mayer U, Meneguzzi G, Miner JH, Miyazaki K, Patarroyo M, Paulsson M, Quaranta V, Sanes JR, Sasaki T, Sekiguchi K, Sorokin LM, Talts JF, Tryggvason K, Uitto J, Virtanen I, von der Mark K, Wewer UM, Yamada Y, Yurchenco PD: A simplified laminin nomenclature. Matrix Biol 2005, 24:326-332

14. Kvist AJ, Nystrom A, Hultenby K, Sasaki T, Talts JF, Aspberg A: The major basement membrane components localize to the chondrocyte pericellular matrix: a cartilage basement membrane equivalent? Matrix Biol 2008, 27:22-33

15. Kruegel J, Sadowski B, Miosge N: Nidogen-1 and nidogen-2 in healthy human cartilage and in late-stage osteoarthritis cartilage. Arthritis Rheum 2008, 58:1422-1432

16. Smyth N, Vatansever HS, Murray P, Meyer M, Frie C, Paulsson M, Edgar D: Absence of basement membranes after targeting the LAMC1 gene results in embryonic lethality due to failure of endoderm differentiation. J Cell Biol 1999, 144:151-160

17. Thomas T, Dziadek M: Genes coding for basement membrane glycoproteins laminin, nidogen, and collagen IV are differentially expressed in the nervous system and by epithelial, endothelial, and mesenchymal cells of the mouse embryo. Exp Cell Res 1993, 208: 54-67

18. Pritzker KP, Gay S, Jimenez SA, Ostergaard K, Pelletier JP, Revell PA, Salter D, van den Berg WB: Osteoarthritis cartilage histopathology: grading and staging. Osteoarthritis Cartilage 2006, 14: $13-29$

19. Altman R, Asch E, Bloch D, Bole G, Borenstein D, Brandt K, et al; Diagnostic and Therapeutic Criteria Committee of the American Rheumatism Association: Development of criteria for the classification and reporting of osteoarthritis: classification of osteoarthritis of the knee. Arthritis Rheum 1986, 29:1039-1049

20. Schymeinsky J, Nedbal S, Miosge N, Poschl E, Rao C, Beier DR, Skarnes WC, Timpl R, Bader BL: Gene structure and functional analysis of the mouse nidogen-2 gene: nidogen-2 is not essential for basement membrane formation in mice. Mol Cell Biol 2002, 22 : 6820-6830

21. Glasson SS, Chambers MG, Van Den Berg WB, Little CB: The OARSI histopathology initiative: recommendations for histological assessments of osteoarthritis in the mouse. Osteoarthritis Cartilage 2010, 18(Suppl 3):S17-S23

22. Miosge N, Gunther E, Heyder E, Manshausen B, Herken R: Light and electron microscopic localization of the alpha 1-chain and the E1 and E8 domains of laminin-1 in mouse kidney using monoclonal antibodies to establish the orientation of laminin-1 within basement membranes. J Histochem Cytochem 1995, 43:675-680

23. Holtzer H, Abbott J, Lash J, Holtzer S: The loss of phenotypic traits by differentiated cells in vitro, I: dedifferentiation of cartilage cells. Proc Natl Acad Sci U S A 1960, 46:1533-1542

24. Pfaffl MW: A new mathematical model for relative quantification in real-time RT-PCR. Nucleic Acids Res 2001, 29:e45

25. Christian H, Hofele RV, Urlaub H, Ficner R: Insights into the activation of the helicase Prp43 by biochemical studies and structural mass spectrometry. Nucleic Acids Res 2014, 42:1162-1179

26. Goldring MB, Goldring SR: Articular cartilage and subchondral bone in the pathogenesis of osteoarthritis. Ann N Y Acad Sci 2010, 1192: 230-237

27. Ali SY, Griffiths S: Formation of calcium phosphate crystals in normal and osteoarthritic cartilage. Ann Rheum Dis 1983, 42(Suppl 1):45-48

28. Yahata T, de Caestecker MP, Lechleider RJ, Andriole S, Roberts AB, Isselbacher KJ, Shioda T: The MSG1 non-DNA-binding transactivator binds to the $\mathrm{p} 300 / \mathrm{CBP}$ coactivators, enhancing their functional link to the Smad transcription factors. J Biol Chem 2000, 275:8825-8834
29. Cucchiarini M, Thurn T, Weimer A, Kohn D, Terwilliger EF, Madry H: Restoration of the extracellular matrix in human osteoarthritic articular cartilage by overexpression of the transcription factor SOX9. Arthritis Rheum 2007, 56:158-167

30. Goldwasser M, Astley T, van der Rest M, Glorieux FH: Analysis of the type of collagen present in osteoarthritic human cartilage. Clin Orthop Relat Res 1982, (167):296-302

31. Wang X, Manner PA, Horner A, Shum L, Tuan RS, Nuckolls GH: Regulation of MMP-13 expression by RUNX2 and FGF2 in osteoarthritic cartilage. Osteoarthritis Cartilage 2004, 12:963-973

32. Kamekura S, Kawasaki Y, Hoshi K, Shimoaka T, Chikuda H, Maruyama Z, Komori T, Sato S, Takeda S, Karsenty G, Nakamura K, Chung UI, Kawaguchi H: Contribution of runt-related transcription factor 2 to the pathogenesis of osteoarthritis in mice after induction of knee joint instability. Arthritis Rheum 2006, 54:2462-2470

33. Mokkapati S, Bechtel M, Reibetanz M, Miosge N, Nischt R: Absence of the basement membrane component nidogen 2, but not of nidogen 1 , results in increased lung metastasis in mice. J Histochem Cytochem 2012, 60:280-289

34. Amann K, Haas CS, Zeiler GA, Benz K, Bader BL, Hartner A, Hilgers KF: Lack of nidogen-2 increases blood pressure, glomerular and tubulointerstitial damage in DOCA-salt hypertension. Eur J Clin Invest 2009, 39:116-125

35. Maegdefrau U, Bosserhoff AK: BMP activated Smad signaling strongly promotes migration and invasion of hepatocellular carcinoma cells. Exp Mol Pathol 2012, 92:74-81

36. Murakami S, Kan M, McKeehan WL, de Crombrugghe B: Upregulation of the chondrogenic Sox 9 gene by fibroblast growth factors is mediated by the mitogen-activated protein kinase pathway. Proc Natl Acad Sci U S A 2000, 97:1113-1118

37. Watt FM, Hogan BL: Out of Eden: stem cells and their niches. Science 2000, 287:1427-1430

38. Toh WS, Foldager CB, Olsen BR, Spector M: Basement membrane molecule expression attendant to chondrogenesis by nucleus pulposus cells and mesenchymal stem cells. J Orthop Res 2013, 31:1136-1143

39. Fuchs E, Tumbar T, Guasch G: Socializing with the neighbors: stem cells and their niche. Cell 2004, 116:769-778

40. Schlotzer-Schrehardt U, Dietrich T, Saito K, Sorokin L, Sasaki T, Paulsson M, Kruse FE: Characterization of extracellular matrix components in the limbal epithelial stem cell compartment. Exp Eye Res 2007, 85:845-860

41. Suh HN, Kim MO, Han HJ: Laminin-111 stimulates proliferation of mouse embryonic stem cells through a reduction of gap junctional intercellular communication via RhoA-mediated $\mathrm{Cx} 43$ phosphorylation and dissociation of Cx43/ZO-1/drebrin complex. Stem Cells Dev 2012, 21:2058-2070

42. Rooney JE, Knapp JR, Hodges BL, Wuebbles RD, Burkin DJ: Laminin-111 protein therapy reduces muscle pathology and improves viability of a mouse model of merosin-deficient congenital muscular dystrophy. Am J Pathol 2012, 180:1593-1602

43. Nguyen-Ngoc KV, Cheung KJ, Brenot A, Shamir ER, Gray RS, Hines WC, Yaswen P, Werb Z, Ewald AJ: ECM microenvironment regulates collective migration and local dissemination in normal and malignant mammary epithelium. Proc Natl Acad Sci U S A 2012, 109:E2595-E2604

44. Gorfu G, Virtanen I, Hukkanen M, Lehto VP, Rousselle P, Kenne E, Lindbom L, Kramer R, Tryggvason K, Patarroyo M: Laminin isoforms of lymph nodes and predominant role of alpha5-laminin(s) in adhesion and migration of blood lymphocytes. J Leukoc Biol 2008, 84:701-712

45. Hashimoto K, Otero M, Imagawa K, de Andres MC, Coico JM, Roach HI, Oreffo RO, Marcu KB, Goldring MB: Regulated transcription of human matrix metalloproteinase 13 (MMP13) and interleukin-1beta (IL1B) genes in chondrocytes depends on methylation of specific proximal promoter CpG sites. J Biol Chem 2013, 288:10061-10072

46. Fuerst FC, Gruber G, Stradner MH, Jones JC, Kremser ML, Angerer H, Setznagl D, Glehr M, Windhager R, Leithner A, Graninger WB: Regulation of MMP3 by laminin alpha 4 in human osteoarthritic cartilage. Scand J Rheumatol 2011, 40:494-496 